\title{
Seeded Supercontinuum Generation - Modulation Instability Gain, Coherent and Incoherent Rogue Waves
}

Sørensen, Simon Toft; Larsen, Casper; Møller, Uffe Visbech; Moselund, P. M.; Thomsen, C. L.; Bang, Ole

Published in:

Proceedings of Frontiers in Optics (FiO)

Publication date:

2012

Document Version

Publisher's PDF, also known as Version of record

Link back to DTU Orbit

Citation (APA):

Sørensen, S. T., Larsen, C., Møller, U. V., Moselund, P. M., Thomsen, C. L., \& Bang, O. (2012). Seeded Supercontinuum Generation - Modulation Instability Gain, Coherent and Incoherent Rogue Waves. In Proceedings of Frontiers in Optics (FiO) (pp. FTh1D.4). Optical Society of America.

\section{General rights}

Copyright and moral rights for the publications made accessible in the public portal are retained by the authors and/or other copyright owners and it is a condition of accessing publications that users recognise and abide by the legal requirements associated with these rights.

- Users may download and print one copy of any publication from the public portal for the purpose of private study or research.

- You may not further distribute the material or use it for any profit-making activity or commercial gain

- You may freely distribute the URL identifying the publication in the public portal 


\title{
Seeded Supercontinuum Generation - Modulation Instability Gain, Coherent and Incoherent Rogue Waves
}

\author{
S. T. Sørensen ${ }^{1, *}$, C. Larsen ${ }^{1}$, U. Møller ${ }^{1}$, P. M. Moselund ${ }^{2}$, C. L. Thomsen ${ }^{2}$, and O. Bang ${ }^{1,2}$ \\ ${ }^{I}$ DTU Fotonik, Department of Photonics Engineering, Technical University of Denmark, 2800 Kgs. Lyngby, Denmark \\ ${ }^{2}$ NKT Photonics A/S, Blokken 84, DK-3460, Birkerød, Denmark \\ ${ }^{*}$ Corresponding author: $\underline{\text { stso@ fotonik.dtu.dk }}$
}

\begin{abstract}
Deterministic supercontinuum can be generated by seeding the modulation instabilityinduced pulse break-up. We investigate the influence of the modulation instability gain on seeding and demonstrate the generation of coherent and incoherent rogue waves.

OCIS codes: (190.4380) Nonlinear optics, four-wave mixing; (320.6629) Supercontinuum generation.
\end{abstract}

\section{Introduction}

The noise properties of a supercontiuum (SC) can be significantly improved both in terms of coherence and intensity stability by modulating the input pulse with a seed [1-2]. In the long pulsed regime, the SC generation is initiated by noise-seeded modulation instability (MI), which breaks the pump into solitons and dispersive waves. To reduce the noise of the SC, it has been proposed to provide a seed, i.e. a weak pulse with a frequency offset relative to the pump, within the MI gain spectrum in order to ensure a deterministic rather than noise-seeded pulse break-up [1]. In this work, we numerically investigate the influence of the seed wavelength, the pump power and the MI gain spectrum on the seeding process. We highlight the general mechanisms and show that the results can be clearly divided into a number of distinct dynamical regimes depending on the initial four-wave mixing process. We further demonstrate that seeding can be used to generate coherent and incoherent rogue waves.

Based on numerical simulations of the generalized nonlinear Schrödinger equation, we quantify the noise properties of ensemble averages of independent SC spectra. We quantify the noise both by the widely used spectral coherence function and the signal-to-noise ratio (SNR) defined as the mean to the standard deviation calculated of the ensemble.

\section{Results}

We use a fiber with a zero-dispersion wavelength (ZDW) of $1054 \mathrm{~nm}$ and a $3 \mathrm{ps}$ (FWHM) pump with a peak power of $250 \mathrm{~W}$. The seed has the same temporal width but only $5 \%$ of the pump peak power. Typical results of seeding the pulse break-up are seen in Fig. 1(a): When the pump and seed are overlapping (Fig. 1(a1)), the spectral broadening is initiated by noise-seeded MI. The spectrum is only coherent near the pump and has near unity SNR over the entire bandwidth. By shifting the seed $3 \mathrm{THz}$ away from the pump (Fig. 1(a2)), i.e. in the Stokes band, cascaded four-wave mixing (FWM) generates a frequency comb of side-bands with a $3 \mathrm{THz}$ separation. The number of side-bands is limited by the width of the MI gain shown in (Fig. 1(a4)). The resulting spectrum is coherent over most of the bandwidth and has improved SNR. When the seed is shifted further away from the pump to $13 \mathrm{THz}$ (Fig. 1(a3)), only one set of side-bands is amplified, which leads to the generation of one massive rogue soliton. This is exactly what was referred to as 'Harnessing and control of optical rogue waves' in [3]. The rogue soliton is generated incoherently but with high intensity stability.

Figure 1(b) shows ensemble averaged results for both the spectrum, coherence and SNR for pump-seed frequency offsets spanning beyond the entire MI gain bandwidth. There is almost perfect mirror-symmetry around zero frequency offset, and we only discuss seeding the Stokes band. For small frequency offsets $(<5 \mathrm{THz})$, cascaded FWM with many peaks leads to a coherent pulse breakup and a spectrum with high coherence and SNR. When the frequency offset is increased, the number of amplified peaks decreases and the coherence of the central part of the spectrum degrades. For frequency offsets of $\sim 10-20 \mathrm{THz}$ only a single set of side-bands are amplified via FWM and a large amplitude soliton is generated. The spectrum is only coherent near the residual pump and FWM side-bands, but the soliton is intensity stable. In a frequency range around $\sim 20 \mathrm{THz}$ no large amplitude soliton is ejected, but the FWM side-bands undergo self-phase modulation (SPM) and broaden coherently with high intensity stability. In this regime, the coherence improves when the frequency offset approaches the MI gain peak. This growth has not been clearly observed before. When the seed is shifted outside the MI gain spectrum, the spectrum is largely incoherent except near the weak FWM side-bands. The coherence plot further shows a high degree of coherence near the higher-order side-bands. 
(a) Single simulations

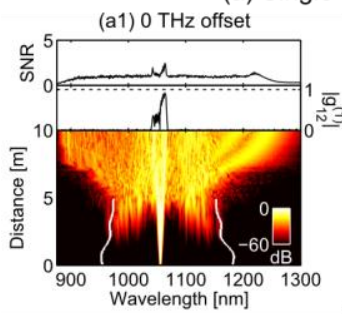

(a3) $13 \mathrm{THz}$ offset

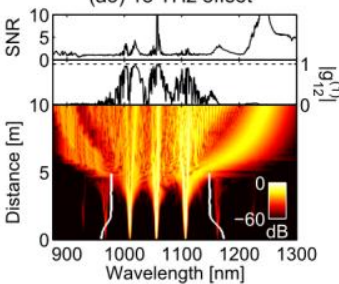

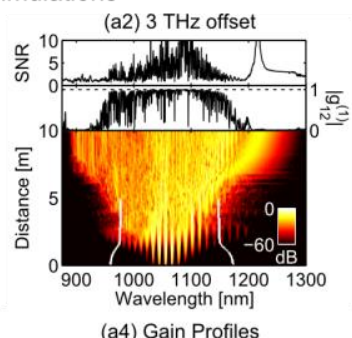

(a4) Gain Profiles

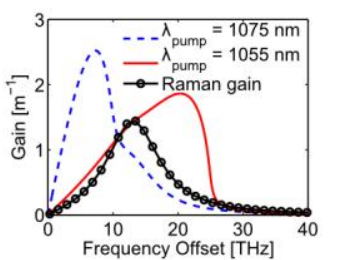

(b) Ensemble averaged results (b2) Coherence
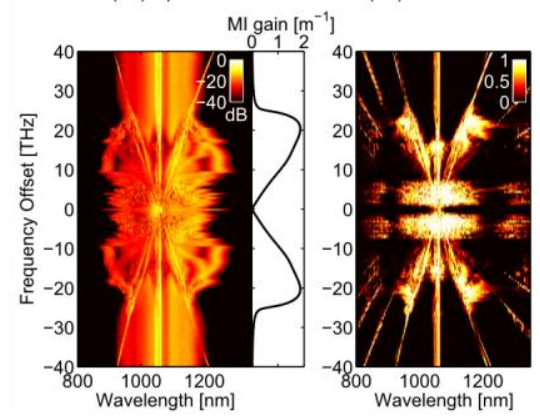

(b3) Signal-to-noise ratio

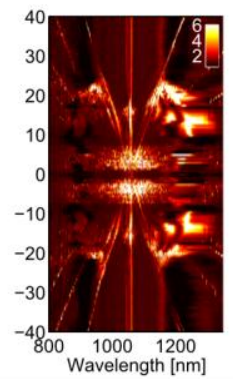

Fig. 1: (a) Single-shot simulations of pumping at $1055 \mathrm{~nm}$ with a 3 ps (FWHM) Gaussian pump with a $250 \mathrm{~W}$ peak power, and a seed with the same width and $5 \%$ of the peak power at frequency offsets of (a1)-(a3) 0, 3 and $13 \mathrm{THz}$. The white lines show the MI gain bandwidth. (a4) MI gain profiles for pump wavelengths of 1075 and $1055 \mathrm{~nm}$, and the pure Raman gain. (b) Pseudo color plots of the ensemble averaged (b1) spectra and calculated (b2) coherence and (b3) SNR for the same parameters as in (a). 200 simulations are used in the calculations.

The MI gain spectrum narrows when the pump is moved further away from the ZDW, as shown in Fig. 1(a4). This difference is illustrated in Fig. 2, where the temporal evolution and spectrograms at the fiber output are shown for pump wavelengths of 1055 and $1075 \mathrm{~nm}$. The seed causes a beating of the temporal profile, which leads to a deterministic pulse breakup. When the pump is close to the ZDW (Fig. 2(a)), the MI gain is small and slowly increasing with frequency. The temporal profile is therefore only slowly broken up into solitons. This means that the solitons are mainly generated from the pulse center where the peak power is highest. The solitons have time to redshift before the cascade is amplified and the dynamics are relatively turbulent. In contrast to this, pumping further from the ZDW (Fig. 2(b)) gives a much larger and more rapidly increasing gain. This causes a fast breakup of the temporal pulse, where the individual temporal fringes generate fundamental solitons in a controlled fashion that almost resembles soliton fission. The most powerful solitons are still generated near the center of the pulse where the power is highest. These powerful solitons only collide with the smaller solitons generated from the trailing edge of the pulse. Interestingly, it turns out that the rogue soliton is generated incoherently when pumping close to the ZDW (as seen in Fig. 1(a1)), but completely coherently when the pump is shifted away from the ZDW (not shown).

(a) $\lambda_{\text {pump }}=1055 \mathrm{~nm}, 4 \mathrm{THz}$ offset

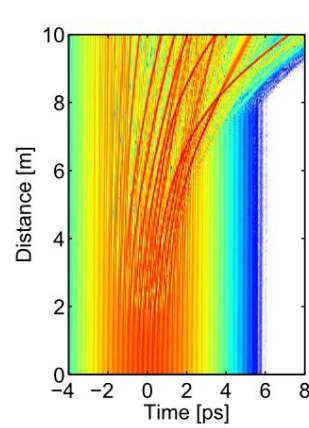

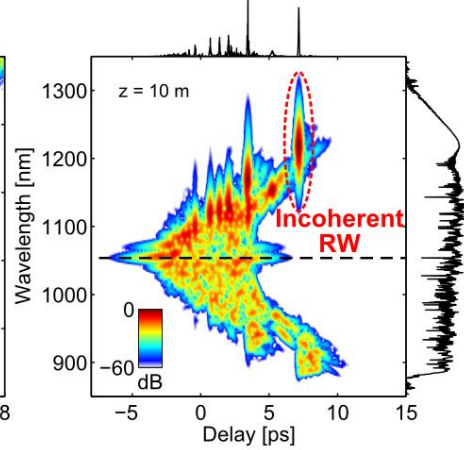

(b) $\lambda_{\text {pump }}=1075 \mathrm{~nm}, 4 \mathrm{THz}$ offset

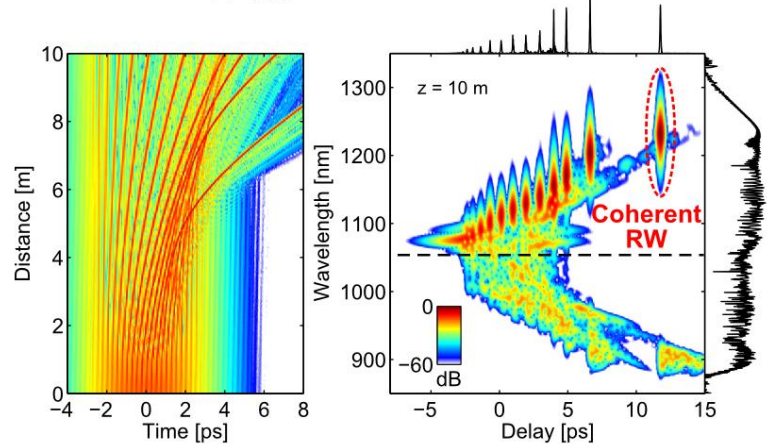

Fig. 2: Temporal evolution and spectrogram at the fiber output (10 m) for a (a) $1055 \mathrm{~nm}$ and (b) $1075 \mathrm{~nm}$ pump and a seed at $4 \mathrm{THz}$ frequency offset. All other parameters as in Fig. 1.The dashed lines mark the ZDW.

At the conference we will discuss the influence of the MI gain spectrum in more detail and demonstrate that the coherent pulse break-up afforded by seeding is washed out by turbulent solitonic dynamics when the pump peak power is increased to the $\mathrm{kW}$ level.

[1] G. Genty, J. Dudley, and B. Eggleton, "Modulation control and spectral shaping of optical fiber supercontinuum generation in the picosecond regime," Applied Physics B: Lasers and Optics 94, 187-194 (2009).

[2] D. R. Solli, C. Ropers, and B. Jalali, "Active control of rogue waves for stimulated supercontinuum generation," PRL 101, 233902 (2008).

[3] J. M. Dudley, G. Genty, and B. J. Eggleton, "Harnessing and control of optical rogue waves in supercontinuum generation," Opt. Express 16, 3644-3651 (2008). 